\title{
Evaluation of a low-cost approach to 2-D digital image correlation vs. a commercial stereo-DIC system in Brazilian testing of soil specimens
}

\author{
M. Arza-García ${ }^{1}$ (1) C. Núñez-Temes ${ }^{1}$ · J. A. Lorenzana ${ }^{2} \cdot$ J. Ortiz-Sanz ${ }^{1} \cdot$ A. Castro ${ }^{1} \cdot$ M. Portela-Barral ${ }^{2}$. \\ M. Gil-Docampo ${ }^{1} \cdot$ G. Bastos ${ }^{1}$
}

Received: 21 May 2021 / Revised: 19 September 2021 / Accepted: 11 October 2021 / Published online: 5 November 2021

(c) The Author(s) 2021

\begin{abstract}
Due to their cost, high-end commercial 3D-DIC (digital image correlation) systems are still inaccessible for many laboratories or small factories interested in lab testing materials. These professional systems can provide reliable and rapid full-field measurements that are essential in some laboratory tests with high-strain rate events or high dynamic loading. However, in many stress-controlled experiments, such as the Brazilian tensile strength (BTS) test of compacted soils, samples are usually large and fail within a timeframe of several minutes. In those cases, alternative low-cost methods could be successfully used instead of commercial systems. This paper proposes a methodology to apply 2D-DIC techniques using consumer-grade cameras and the open-source image processing software DICe (Sandia National Lab) for monitoring the standardized BTS test. Unlike most previous studies that theoretically estimate systematic errors or use local measures from strain gauges for accuracy assessment, we propose a contrast methodology with independent full-field measures. The displacement fields obtained with the low-cost system are benchmarked with the professional stereo-DIC system Aramis-3D (GOM GmbH) in four BTS experiments using compacted soil specimens. Both approaches proved to be valid tools for obtaining full-field measurements and showing the sequence of crack initiation, propagation and termination in the BTS, constituting reliable alternatives to traditional strain gauges. Mean deviations obtained between the low-cost 2D-DIC approach and Aramis-3D in measuring in-plane components were $0.08 \mathrm{~mm}$ in the perpendicular direction of loading $(\Delta \mathrm{X})$ and $0.06 \mathrm{~mm}$ in the loading direction $(\Delta \mathrm{Y})$. The proposed low-cost approach implies considerable savings compared to commercial systems.
\end{abstract}

Keywords DIC · Brazilian tensile strength $\cdot$ Stereo vision $\cdot$ GOM $\cdot$ Camera calibration $\cdot$ Full-field measurements

\section{Introduction}

The Brazilian indirect tensile strength (BTS) test is one of the most commonly applied testing methods for the analysis of brittle elastic materials such as granitic rocks [1,2], sandstone [3, 4], concrete [5, 6], bituminous mixtures [7] and stabilized soil $[8,9]$. Unlike direct methods, the BTS test submits disc-shaped samples to a compression line by

\section{Arza-García}

m.arza@usc.es

1 CIGEO-Civil and Geomatics Research Group, Agroforestry Engineering Department, University of Santiago de Compostela, Higher Polytechnic School, 27002 Lugo, Spain

2 PEMADE-Research Platform on Structural Wood Engineering, Agroforestry Engineering Department, University of Santiago de Compostela, Higher Polytechnic School, 27002 Lugo, Spain loading two diametrically opposing points. In this way, a tensile stress is generated inside the cylindrical specimen until it fails. Failure is assumed to occur at the point of maximum tensile stress which should be located at the centre of the cylindrical specimen, according to simple elasticity theory. However, detailed and accurate measurements of the deformation, strain values and points of failure are often required to obtain insight into the overall mechanical behaviour of the sample.

Traditionally, observation of the material deformation during lab testing was carried out by means of mechanical extensometers or strain gauges attached to the face of the specimen. Gauges are typically placed vertically or horizontally to measure diametral strains [10] or across the crack mouth to monitor the opening displacement [11]. However, some previous studies have noticed significant differences in the strain measurements depending on the gauge size and the technology behind each device (e.g., conventional 
electric gauges, fibre-optic-based gauges) [12]. In recent years, the use of digital image correlation (DIC) for the analysis of the displacement and strain of components in many types of tests has received particular attention [13]. Some studies, such as Sgambitterra et al. [14], proposed reliable approaches using DIC for the evaluation of mechanical properties (Young's modulus, Poisson's ratio and tensile strength) of brittle materials during BTS testing. Other studies focussed on the application of DIC in BTS tests for monitoring crack initiation and propagation [15]. DIC's ability to capture data has led to a wide dissemination of this technique in the validation of BTS numerical models trying to understand the deformation behaviours in the full field [16] or more specifically in the fracture process zone [17]. In contrast with traditional methods, DIC provides a continuous field of displacements across the surface of the specimen without the need for direct contact. The principle of DIC is based on tracking the deformation of a random pattern over the surface of the sample during the test using a series of images taken by one camera. The first image, normally taken before loading, is chosen as the reference image and serves as the origin for the displacements in the subsequent deformed images. Then, an automatic procedure of template matching is applied by the processing software in such a way that a small reference region of the pattern (i.e., a correlation window), often called a subset or facet, is identified in both the reference and deformed images [18]. In this simplest case, only two dimensions of displacements can be calculated, and the strains of the material are assumed to be constrained to a plane parallel to the focal plane, as images from just one camera have been used (2D-DIC).

Consumer-grade cameras have been widely used instead of industrial cameras in different applications of two-dimensional DIC, including lab testing of different materials [19, 20] and in-field applications at full scale in large structures such as walls [21] or bridges [22]. Quanjin et al. [23] implemented a low-cost approach of 2D-DIC using consumergrade DSLR cameras and compared the results with strain gauges and with an experimental method. They obtained reasonably good agreement between them. $\mathrm{Li}$ et al. [24] also demonstrated the application of low-cost 2D-DIC with consumer-grade DSLR cameras to determine the thermal expansion coefficients of materials, achieving accuracies comparable to industrial equipment. Zhao et al. [25] investigated the potential of low-cost 2D-DIC to analyse plastic shrinkage cracking on fresh concrete, where traditional methods of contact are not viable. However, it is also possible to estimate three-dimensional deformations using two calibrated parallax cameras (stereo-DIC or 3D-DIC), thus enabling measurement of out-of-plane displacements [26, 27]. This approach requires two perfectly calibrated and synchronized cameras, which makes its application impractical most of the time unless commercial systems with specific binocular
3D systems are used [28]. Constructing a regular 3D-DIC system using two amateur cameras has some complexity, mainly because of the difficulty in accurately performing the internal and external orientations (which requires precise stereo calibration) and because of the synchronization of the cameras. A common way to obtain synchronized photos has been the use of mirror systems or optical prisms to capture in a single image the right and left perspective of the object during the test [29]. This methodology has been successfully tested in performing different deformation measurements with a single DSLR camera [30] and even using a single smartphone [31] with an optical attachment composed of mirrors. Despite this, 3D-DIC techniques using a single camera are generally limited to small specimens with a size of a few tens of millimetres; otherwise, the overlap between diffracted or refracted images leads to decreased image quality [32].

There are a vast number of software packages and libraries, both commercial and open-source solutions that implement optical measurement techniques based on 2D-DIC or 3D-DIC to compute displacements from an image series. Commercial systems, such as Aramis (GOM GmbH), StrainMaster (LaVision), Istra4D (Dantec Dynamics) and VIC-3D (Correlated Solutions), are also generally provided with their own specialized hardware and optical components (e.g., ultrahigh speed cameras for DIC, calibration plates, illumination systems). Although these high-end systems constitute ready-to-use and very reliable solutions, their main limitation is their inherent cost. Alternatively, the scientific community makes available to users several open-source DIC programmes that can be useful for many laboratories, small factories, or research groups with more limited resources (e.g., MultiDIC [33], Ncorr [34], $\mu$ DIC [35], py2DIC [36]). Some of these programmes are implemented in the MAT$\mathrm{LAB}$ language, so it is also necessary to purchase a licence [37]. Fortunately, there is also space for open-source solutions such as DICe (SNL, Albuquerque, USA) that can be directly run on most operating systems. Although the range of options for the user is varied, it is still difficult to make comparisons with DIC from separate studies, as the analysis parameters are addressed differently by each software package and even for the same test and using the same images, the final strain maps may look markedly different [38].

Previous studies have quantitatively evaluated the results of free 2D-DIC software processing, some of them even in combination with amateur cameras. However, the precision assessment of DIC systems is frequently based on theoretical estimation of systematic errors in local measurements or simply on the determination of the noise floor. Few of the studies have set reference measures from which to quantify the errors, except direct measures from strain gauges. The problem is that the strain field gradients that occur during loading are hardly comparable with the local reads of 
strain gauges. Thus, an accuracy contrast methodology with independent full-field measures would be desirable in those cases.

This article describes a procedure to perform full-field measurements of displacements during BTS testing using a 2D-DIC system that combines consumer-grade cameras and open-source software (DICe). The aim is to investigate a reliable low-cost alternative for small labs to avoid the need to acquire commercial DIC equipment. A total of four BTS tests were performed to analyse the potential of the proposed low-cost approach in monitoring compacted soil specimens during the standardized Brazilian test. The high-end commercial 3D-DIC system Aramis 3D, which integrates its own industrial cameras $(12 \mathrm{M})$ with a professional stereo-DIC processing module, was used to benchmark the performance and the resulting full-field measurements of the low-cost system in terms of accuracy. It is important to emphasize that this is not just a comparison of software but of the systems as a whole (i.e., optical systems and processing approaches); therefore, each system was used with its own images. Based on the performed experiments, general recommendations for conducting measurements in BTS testing are provided.

\section{Methodology}

\subsection{BTS testing setup}

There are several standardized procedures for applying the BTS test to the measurement of tensile strength, but the most commonly used approaches are the ISRM [39] and ASTM [40], both of which outline direct and indirect Brazilian test methods. Whilst traditionally the use of flat loading platens was adopted for BTS testing, further studies suggested that curved platens could be more appropriate, just to mitigate the crushing effect of the loaded points, as the elastic properties did not suffer in principle any influence from the loading configuration [14, 41]. Therefore, the tests were performed by applying a compressive load at two points using curved breaking heads specifically designed to adapt the sample dimensions, thus preventing local compressive failure without significantly altering the tensile stress conditions. Although the loading rate has not previously been shown to have a significant effect on measured tensile stresses, it is assumed that lower ratios (i.e., $<1 \mathrm{~mm} \mathrm{~min}{ }^{-1}$ ) produce greater repeatability than higher ratios $[8,9]$. To achieve failure within a reasonable timeframe (within 1 to $10 \mathrm{~min}$ ), the loading in the two experiments performed here was applied with a gradient of $0.2-0.5 \mathrm{~mm} \mathrm{~min}^{-1}$. A motorized load frame S205N (MATEST S.p.A., Treviolo, Italy) was used to apply these constant rates of loading.

The soil specimens were prepared according to the procedure established in the UNE-13286:2011 [42] standard for the preparation of soil samples stabilized with hydraulic binders in laboratory testing. The specimens were compacted following the Proctor modified test procedure using a type $\mathrm{B}$ mould of known volume, with a compaction energy of 2,586 $\mathrm{MJ} \mathrm{m}^{-3}$ and an optimum moisture content determined from previous tests. Once compacted, the samples remain in the mould for $24 \mathrm{~h}$ in a humidity chamber for an adequate curing process. After this period, they are removed from the mould and stored until the test date under stable conditions of humidity $(>90 \%)$ and temperature $\left(20^{\circ} \mathrm{C}\right)$ in the humidity chamber.

The tests described in Sect. 3 were carried out using four different specimens: the first was composed of silty soil (ML in USCS classification) stabilized with $4 \%$ cement, and the other three specimens had an additional stabilizer (i.e., $4 \%$ of a biowaste rich in lignin obtained from the wood panel industry) [43]. The biowaste employed was a viscous, brown liquid rich in microfibres obtained by partial liquefaction of eucalyptus wood powder in deep eutectic solvents (DESs) and a subsequent process of decantation. The resulting product was a mixture of $26 \%$ pure lignin (16.5\% Klaxon lignin/9.47\% soluble lignin) with ashes and sugars. Whilst the aim of the research is not to analyse the composition of testing samples, the potential application of this type of industrial biowaste in soil stabilization is currently generating great interest $[44,45]$. The properties of these specimens at the time of their elaboration are shown in Table 1.

\subsection{DIC testing setup}

The final accuracy of DIC full-field measurements mainly relies on the capacity of the software algorithms to precisely identify and track the displacement of small parts of the

Table 1 Characteristics of the compacted soil specimens employed in the BTS testing

\begin{tabular}{lllllcr}
\hline Specimen description & $\begin{array}{l}\text { Avg. diameter } \\
(\mathrm{mm})\end{array}$ & Avg. height $(\mathrm{mm})$ & Total mass $(\mathrm{g})$ & Volume $\left(\mathrm{cm}^{3}\right)$ & $\begin{array}{c}\text { Moisture }(\%) \\
\left(\mathrm{g} \cdot \mathrm{cm}^{-3}\right)\end{array}$ \\
\hline BTS-S1 4\% CEM & 152.8 & 128.0 & 4878 & 2347.18 & 15.2 & 1.804 \\
BTS-S2 4\% CEM 4\% LIG & 151.9 & 128.3 & 4812 & 2325.05 & 16.4 & 1.778 \\
BTS-S3 4\% CEM 4\% LIG & 152.3 & 128.0 & 4655 & 2331.84 & 19.0 & 1.678 \\
BTS-S4 4\% CEM 4\% LIG & 152.0 & 127.2 & 4764 & 2308.15 & 17.6 & 1.755 \\
\hline
\end{tabular}


image along the sequence of images. For that reason, the quality of the object texture is crucial to achieve an accurate match between the elements of the reference (first) image and the same elements in the deformed ones. Although some materials, such as natural rocks or concrete, may exhibit an inherent speckled pattern [46], the texture of the compacted soil specimens does not generally contain enough identifiable features. In those cases, the greyscale values of the images may not be sufficiently stable to capture small incremental deformations. Therefore, a black-on-white random speckle pattern (Fig. 1) was applied as uniformly as possible, using opaque spray paint on the plain face of each sample. A rigid paint film over the face may modify the mechanical stress conditions in the sample, so it is very advisable to use
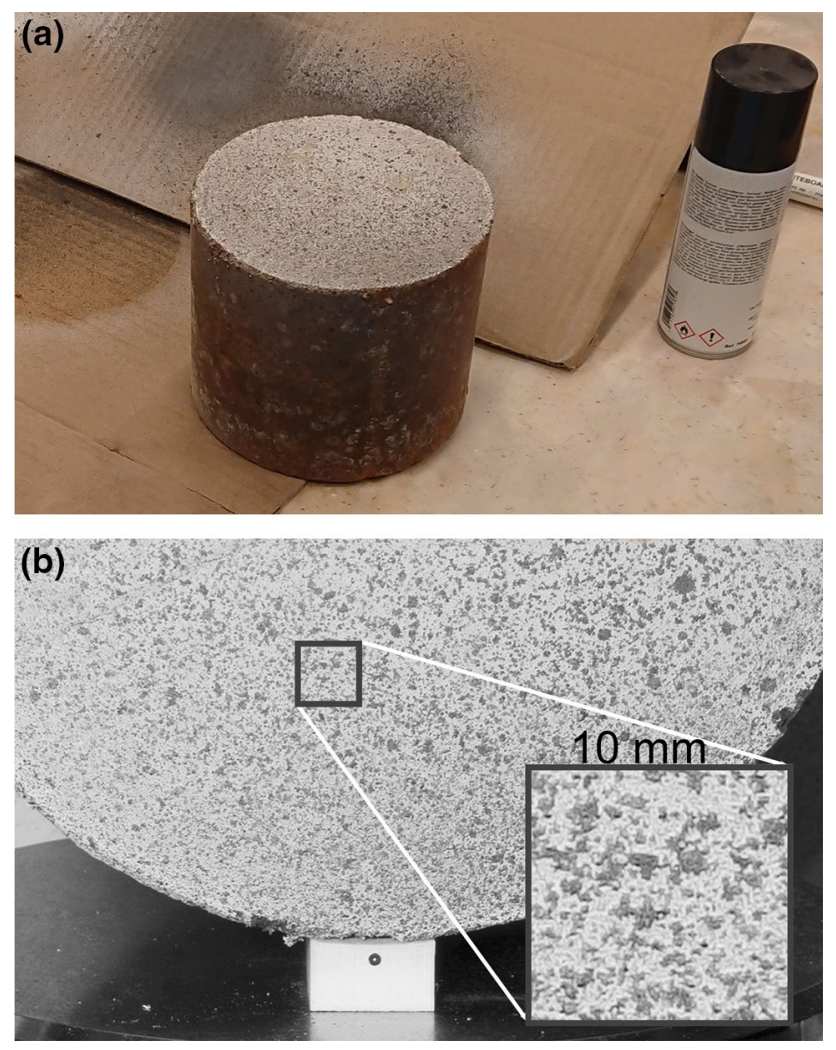

Fig. 1 Detail of the speckled pattern: the application of a fine aerosol white coating followed by a spot distribution of black paint applied to specimens and the resulting $\mathbf{b}$ isotropic speckled pattern with an approximate $25-40 \%$ coverage special paints for DIC that adhere to the surface but deform easily with it. Thus, a professional acrylic spray enamel (Top Acrylic RAL ref. 9005 and 9010) recommended by the GOM's dealer was used (format $400 \mathrm{ml}$ aerosol, drying 30-60 min, dry film thickness $13-15$ microns according to ISO 2808 and gloss 5-10 GE (matt) according to DIN 67,530).

The experimental DIC setup was carefully designed to simultaneously monitor the BTS tests with the stereo-DIC and the 2D-DIC low-cost system to make comparisons. A Sony ILCE 6000 mirrorless camera (tests BTS-S1 and S2) and a DSLR Canon EOS550D (tests BTS-S3 and S4) were used in the tests, whilst data were simultaneously captured with Aramis 3D ${ }^{\circledR}$ (GOM mbH, Braunschweig, Germany) (Table 2). As 2D-DIC requires that the surface of the specimen be positioned perpendicular to the axis of the camera, the optical module of Aramis (composed of two $12 \mathrm{M}$ industrial cameras) was placed in an oblique position, which also allows it to record out-of-plan deformations. The placement of the Aramis optical module was also conditioned by the GOM's recommended distance to the specimen, varying according to the calibration template used (in this case $295 \mathrm{~mm}$ ) with an angle of $25.2^{\circ}$ between cameras (typically, the stereo-angle should be between approximately $15^{\circ}-35^{\circ}$ [47]). Despite being in an oblique position, the entire front face of the sample falls into the measuring volume estimated by Aramis in the calibration $(195 \times 160 \times 150 \mathrm{~mm}$ of weight $\times$ height $\times$ field, respectively), ensuring that the depth of field is large enough to have adequate focus over the surface of the specimen. In a similar way, the setup parameters of the consumer-grade cameras [i.e., focal length, setup distance and field of view (FoV)] were selected together because all of them are intertwined and related to other parameters that are intrinsic to the camera (e.g., CCD size). Cameras and lenses cannot be selected independently due to the combined sensor size and lens effect on the resulting image scale. However, the position and setup of the cameras should be prioritized for matching the desired FoV to the desired area of interest (AOI) [48]. Moreover, there are certain rules of thumb that should be considered in 2D-DIC, such as avoiding very small focal lengths $\left(<15^{\circ}\right)$, to maximize the distance to the object and hence minimize errors caused by out-of-plane motion. With this setup (Fig. 2), both DIC systems were confirmed to meet the overall recommendation
Table 2 Camera specifications and setup parameters

\begin{tabular}{lllll}
\hline Camera & CCD Size (pixels) & Pixels per mm & $\begin{array}{l}\text { Focal } \\
\text { length } \\
(\mathrm{mm})\end{array}$ & FoV (at setup distance) (mm) \\
\hline Sony ILCE 6000 & $6000 \times 4000$ & 255 & 32 & $382 \times 254$ (at 525) \\
Canon EOS 550D & $5196 \times 3464$ & 233 & 20 & $391 \times 261$ (at 350) \\
Aramis 12 M (dual) & $4096 \times 3000$ & 204 & 24 & $200 \times 150($ at 295) \\
\hline
\end{tabular}


Fig. 2 2D and 3D-DIC setup

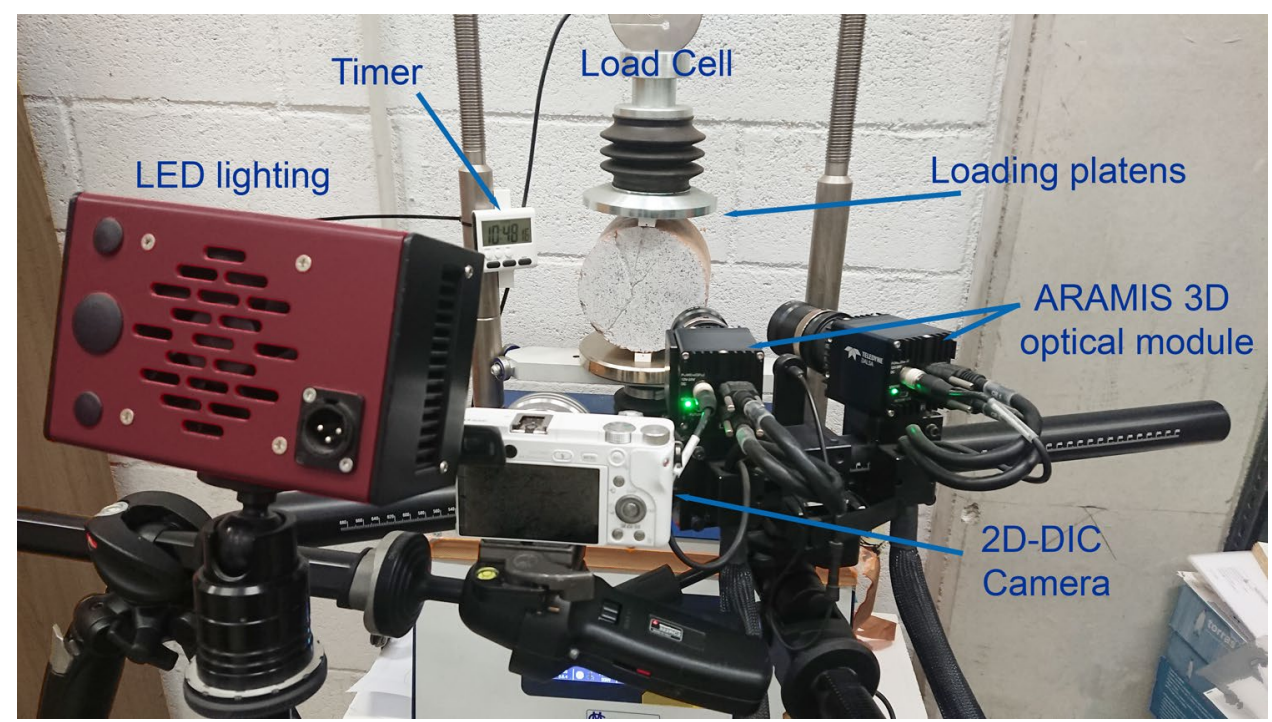

of 2-30 pixels per speckle in the pattern [4]. To ensure an adequate brightness and contrast of the pattern [49], a cold light LED source was also employed. The timers of all the cameras were synchronized prior to the experiments to make a precise adjustment of the image steps in the postprocessing phase. As an additional check measure, a digital millisecond timer whose screen goes into the FoV of the cameras was introduced in the scene.

\subsection{Calibration procedure}

DIC is a methodology with no inherent length scale, as the spatial resolution of the measurements varies with the characteristics of the speckled pattern, the resolution of the images and the established calculation parameters. This fact endows DIC with valuable flexibility to adapt to the study of different types of specimens at different scales but also poses challenges in scaling up the obtained measurements and determining its reliability.

In 2D-DIC, the only theoretical transformation required to convert the pixel space of the programme to real distance units is a scale factor. As this scaling procedure may be based on a single measure, small errors in the distance determination could create large errors in the resulting displacements, so an accurate reference, such as a precision ruler or a calliper, should be used. For this study, a precision ruler was introduced in the scene corresponding to the first (reference) image. The point grid resulting from the DIC processing is then scaled based on a distance measured over this ruler.

In contrast, the positions of the two cameras in 3D-DIC must be calibrated with respect to each other, so a single distance is no longer sufficient for the system calibration. Thus, the common calibration procedure, first proposed by Zhengyou Zhang [50], involves the use of a precision calibration grid (typically a checkerboard or a dotted grid).
The specific Aramis 3D calibration protocol includes an inhouse calibration plate that allows for automatic detection of markers by the software following the same procedure. A set of several images of the calibration plate are obtained under different orientations. The stereo-DIC software uses least squares equations to calculate an accurate mathematical model of the sensor calibration, which includes camera positions and intrinsic parameters (e.g., focal lengths, lens distortions, image centroids). The main benefit of the calibration procedure performed in $3 \mathrm{D}-\mathrm{DIC}$ is that the length scale of the images is accurately connected to the physical length scale of the imaging system. In contrast, the scale of 2D-DIC is introduced by a simple and less accurate conversion between the pixel size of the images and the physical size of the images.

\subsection{Image acquisition and processing}

Image acquisition is, in general, the most important step in DIC, as it is essential to have an appropriate set of images with enough quality to achieve reliable results. Therefore, it is essential to verify that the best-focussed area matches the AOI and to ensure an adequate level of illumination in the image. The consumer-grade cameras were both set with a mid-range aperture of $\mathrm{f} / 7.1$, as the more extreme apertures are expected to introduce more distortions [48], and with an ISO of 100, as increasing the gain increases camera noise. The Aramis software uses an internal protocol based on a calibration plate to correct the diffraction that allows precise adjustment of the aperture size, limiting motion blur and obtaining sufficient contrast. With these settings, parallel images were captured every $2 \mathrm{~s}$ (0.5 fps) with consumergrade cameras and Aramis-3D during the BTS experiments. 
$\mathrm{t}=0 \mathrm{~s}$ (reference) $\quad \mathrm{t}=0 \mathrm{~s}+\mathrm{dt}$ (deformed images)

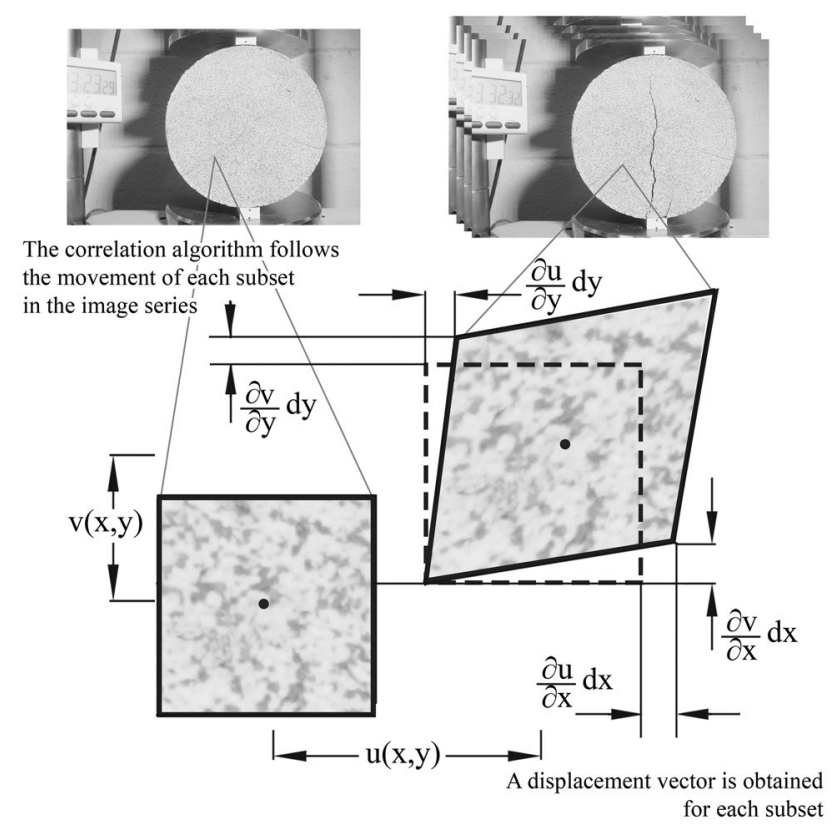

Fig. 3 Fundamentals of 2D-DIC processing

The samples were loaded until the resulting fracture propagated through the whole specimen diameter.

The 2D-DIC images were processed with the open-source software DICe, which is capable of computing full-field displacements and strains from a sequence of images (Fig. 3) [51]. In this case, first-order shape functions, representing translations, rotations, normal strains and shear strains (Eq. 1-2), are used to locate an initial square subset in the reference image within the deformed images [52]:

$\zeta 1=u+\left(\frac{\partial u}{\partial x}\right) \mathrm{dx}+\left(\frac{\partial u}{\partial y}\right) \mathrm{dy}$

$\eta 1=v+\left(\frac{\partial v}{\partial x}\right) \mathrm{dx}+\left(\frac{\partial v}{\partial y}\right) \mathrm{dy}$

where $\zeta 1$ and $\eta 1$ are the total displacements of the subset; $u$ and $v$ are the translations; $\partial \mathrm{u} / \partial \mathrm{x}$ and $\partial \mathrm{v} / \partial \mathrm{y}$ are the normal strains; $\partial \mathrm{u} / \partial \mathrm{y}$ and $\partial \mathrm{v} / \partial \mathrm{x}$ are the shear strains; and $\mathrm{dx}$ and $\mathrm{dy}$ are the distances from the subset centre to an arbitrary point within the same subset in the $\mathrm{x}$ and $\mathrm{y}$ directions, respectively.

The calculation of the displacement maps from 3D-DIC is slightly more complex than from 2D-DIC, as DIC and stereo-vision principles are jointly involved in the transformation between image (2-D) and object space (3-D). Before computing the 3D positions of each grid point, corresponding subsets should be located between the images captured by the two cameras [53]. During this cross-correlation, the perspective transformation from one camera to the other is matched by a shape function defined by the DIC software. This correlation process is equivalent to a $2 \mathrm{D}$-DIC measurement in which the image from one camera (e.g., left) is considered the reference image and the image from the other camera is the deformed image. In addition, as previously mentioned, the stereo calibration protocol provides the parameters needed for the reconstruction of the epipolar geometry, in other words, the intrinsic parameters (principal point, distortion parameters and focal lengths), the extrinsic parameters (camera poses) and the baseline of the stereo camera system [27]. This known stereo geometry constrains the search for homologous points between each pair of photos and allows for improved efficiency and better accuracy in the matching process [54].

Once the corresponding points are located in the right and left images, the 3D position of the point of interest can be computed based on the triangulation principle (Fig. 4). Considering a point $\mathrm{P}$ with its respective projection on each image of the stereo pair $\left[\mathrm{P}_{1}\left(\mathrm{X}_{1}, \mathrm{Y}_{1}\right)\right.$ and $\left.\mathrm{P}_{2}\left(\mathrm{X}_{2}, \mathrm{Y}_{2}\right)\right]$, the $3 \mathrm{D}$ coordinates can be derived from Eq. 3-5. This process can be repeated using a regular grid of points; thus, the 3D shape of the sample in the reference step $(t=0)$ can be obtained for the total AOI.

$X=\frac{B \times \cot \theta 1}{\cot \theta 1+\cot \theta 2}$

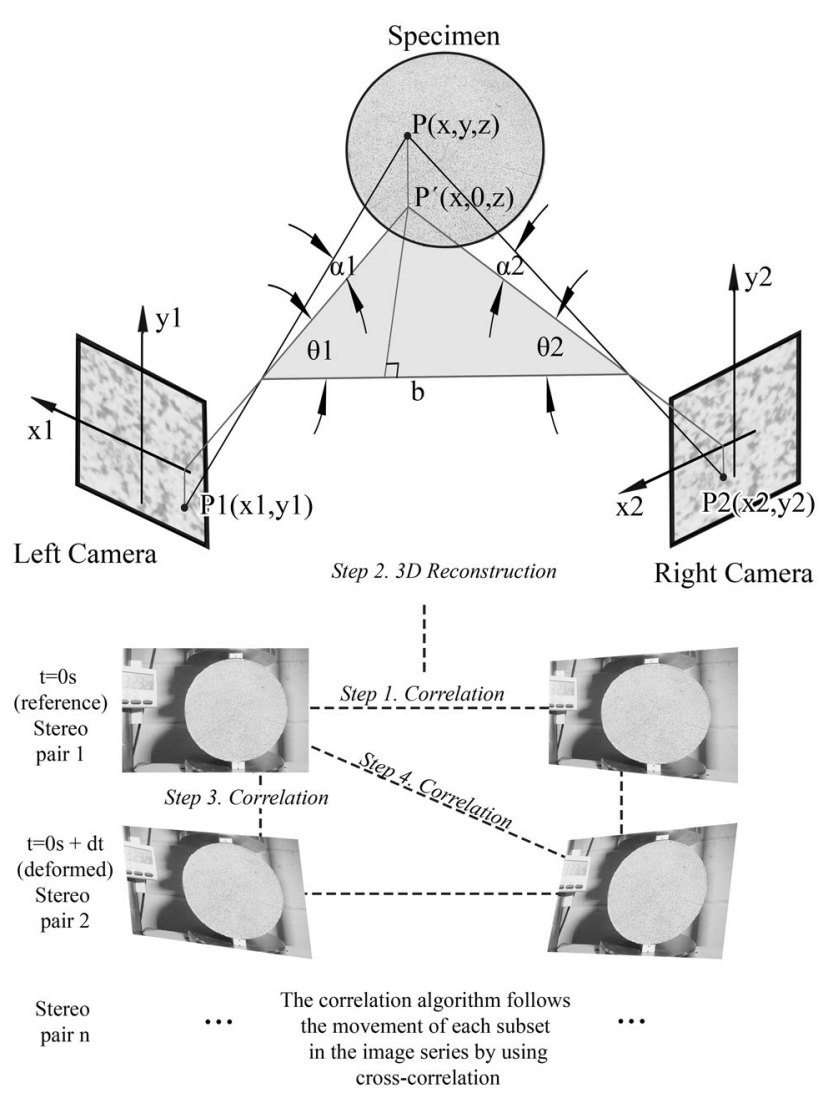

Fig. 4 Fundamentals of stereo-DIC processing 
$Y=\frac{B}{\cot \theta 1+\cot \theta 2} \times \frac{\tan \alpha 1}{\sin \theta 1}$

$Z=\frac{B}{\cot \theta 1+\cot \theta 2}$

The final analysis of 3D displacement vectors and the visualization of the respective maps are based on tracking the movement of the whole set of image subsets between consecutive images of the deformed states. The tracking algorithm uses cross-correlation to find the exact location of the subset in the next image, and the deformation of the subset is considered using the shape function. This process allows us to obtain the full field of displacements with a precision usually assumed to be less than one pixel due to the interpolation of the grey level values between image pixels. A more in-depth mathematical description of the calibration, cross-correlation, and uncertainty quantification, as well as several application examples, can be found in references such as $[55,56]$.

In terms of use, DICe software is very accessible for DIC practitioners and beginners, as it can run directly on the operating system and does not require programming knowledge. Despite performing the calculations, the DICe software does not offer the possibility of directly representing the computed displacement maps and stress fields. An additional application such as Paraview [57] has to be used to open the Exodus (*.e) files for visualization and further processing.

To obtain an optimized balance between the spatial resolution and the interpolation error of the displacement fields, the values of the parameter subset size and step size were manually adjusted. The criterion for setting the subset size value should be to choose a size large enough for a subset to be clearly distinguished from the other subsets. A rule of thumb in DIC is that each subset should contain at least 3 speckles with adequate contrast [48, 58, 59]. Both Aramis and DICe provide a preview of the result before performing calculations so that the user can check whether an increase in size is required to minimize empty areas (poorly correlated subsets in the AOI). On the other hand, the step size or DIC point density defines the distance between subsets, in turn influencing the spatial resolution of the measurements. Step size values are typically lower than subset size. It should be noted that the smaller the step size is, the greater the density of measurements and the longer the calculation time. Nevertheless, a small step size is crucial for studying the differential behaviour of the material in the area near critical elements such as cracks. Following these criteria, values of 29 and 24 pixels were established for the subset size and step size in DICe, respectively, and values of 19 and 16 pixels were used for the corresponding parameters in Aramis. By using equivalent parameters in both systems, the analysis of the results could be simplified, obtaining directly comparable displacement profiles and maps with the same resolution. However, this would not reflect the actual level of accuracy achievable with each of the systems.

The whole front face of the samples $\left(\sim 0.017 \mathrm{~m}^{2}\right)$ was selected as the AOI for both 2D-DIC and 3D-DIC, obtaining an area large enough to ensure sufficient data for qualitative comparison of displacement maps. A group of 50 randomly distributed points along the AOI were selected in both the images of the conventional cameras and those of Aramis to evaluate the results quantitatively. This group of homologous points was used to compare the accuracy of the systems in performing measurements of the two in-plane displacement components and to estimate the error in the 2D-DIC lowcost approach by omitting the out-of-plane movements.

\section{Results and discussion}

The four BTS experiments (S1-S4) were conducted following the general procedures described in the standard UNEEN 13,286-42:2003 [60] but with a modified head of the apparatus to accommodate specific dimensions of the compacted soil specimens, as described in Sect. 2.1. The geotechnical properties of the four samples and BTS test results are summarized in Table 3. In all four experiments, a brittle failure mode was observed as a vertical or near vertical tensile crack, and failure occurred within the first $5 \mathrm{~min}$. The displacement maps were computed for each time step from the dataset of images taken during each of the four tests.

As illustrated in the full-field maps of Fig. 5, the potential of DIC relies on providing a complete distribution of

Table 3 Characteristics and failure behaviour of the tested specimens

\begin{tabular}{|c|c|c|c|c|c|c|}
\hline Specimen description & Age (d) & $\begin{array}{l}\text { Displ. velocity } \\
\left.(\mathrm{mm} \mathrm{min})^{-1}\right)\end{array}$ & $\begin{array}{l}\text { Total mass } \\
\text { change }(\mathrm{g})\end{array}$ & $\begin{array}{l}\text { Tensile strengh } \\
(\mathrm{MPa})\end{array}$ & $\begin{array}{l}\text { Axial Disp. at } \\
\text { failure }(\mathrm{mm})\end{array}$ & Failure strain \\
\hline BTS-S1 4\% CEM & 8 & 0.5 & -6 & 0.151 & 1,529 & 0.010 \\
\hline BTS-S2 4\% CEM 4\% LIG & 8 & 0.5 & -10 & 0.130 & 1,207 & 0.008 \\
\hline BTS-S3 4\% CEM 4\% LIG & 8 & 0.2 & -3 & 0.088 & 1,060 & 0.007 \\
\hline BTS-S4 4\% CEM 4\% LIG & 8 & 0.2 & +9 & 0.086 & 1,159 & 0.008 \\
\hline
\end{tabular}


Fig. 5 Test 1. Displacement maps perpendicular to the loading direction before failure $(t=186 \mathrm{~s})$ with a Aramis and $\mathbf{b}$ DICe and after failure $(t=204 \mathrm{~s})$ with $\mathbf{c}$ Aramis and d DICe. Colour scale equivalence is approximated
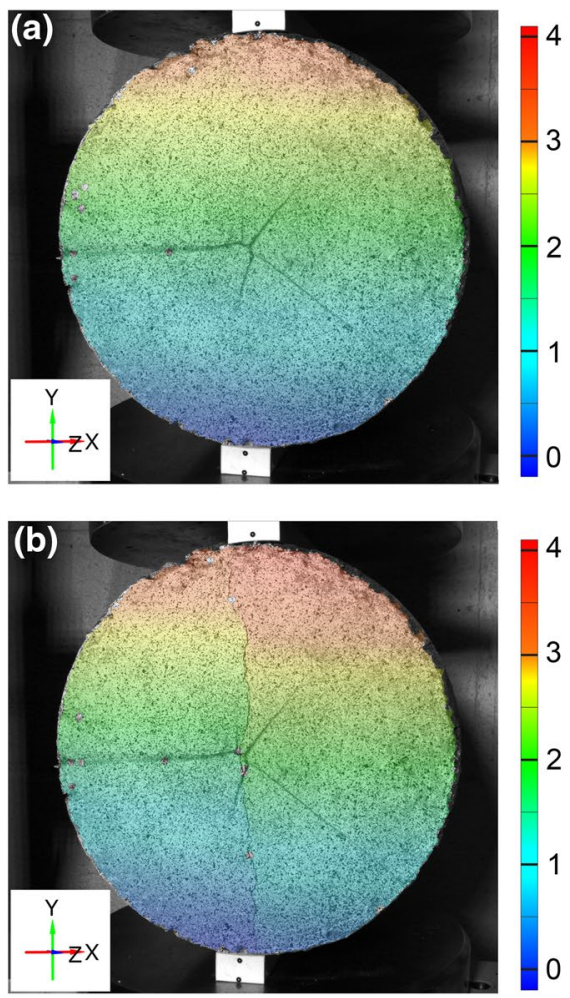

(b)
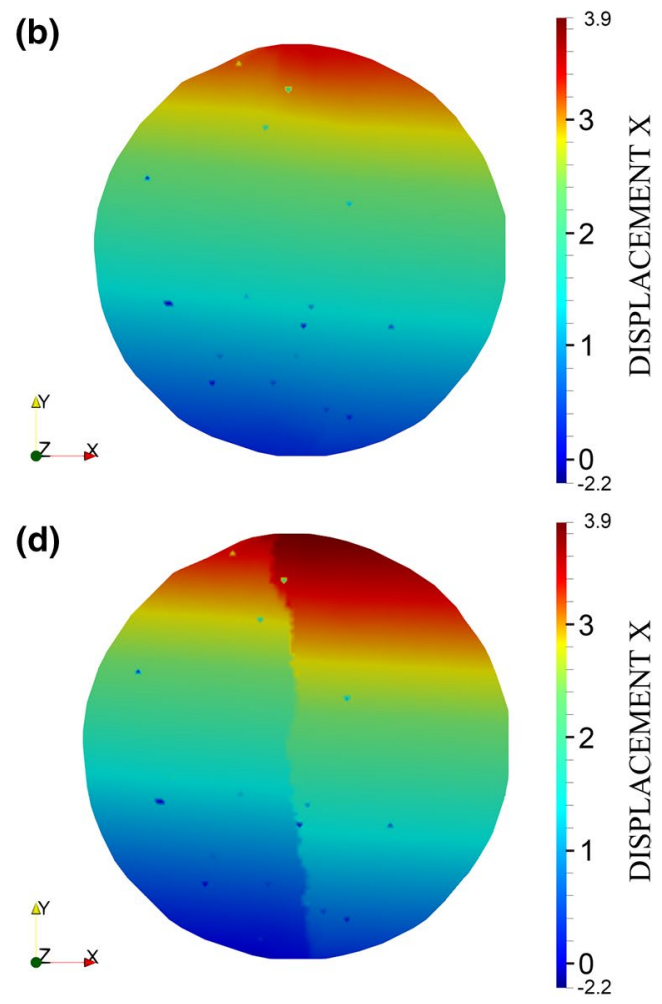

displacement data during the tests, in contrast to strain gauges, which only provide discrete data over an averaged area of application. With the setup and processing parameters established in the previous section, both systems provide in-plane displacement maps that are qualitatively very coincident. These maps allow us to observe the global behaviour of the specimen surface throughout the whole test and to identify local phenomena due to material inhomogeneity and cracking at various levels (i.e., primary/secondary cracks). In that sense, both approaches proved to be valid tools if we considered the requirement of DIC to show the sequence of crack initiation, propagation and termination [61]. Obviously, the exploitation of the results and further analysis options in the commercial software Aramis outperform the low-cost software, which lacks important features, such as the possibility to directly overlay the original image. However, most of these shortcomings can be overcome after postprocessing using other visualization and analysis software such as Paraview. On the other hand, the integration of acquisition and analysis capabilities in a single product ensures that the data gathered with Aramis are handled quickly, being able to compute full-field maps of displacements and strains in almost real time. It is also important to highlight that even when using a conventional DSLR or a compact camera, DIC can produce a large amount of data during long recording times, which will significantly increase the postprocessing time. A couple of videos of the amateur 2D-DIC performance as a method for gathering the displacements during BTS tests are provided in the electronic Supplementary information.

Some correlation artefacts can be observed due to the lower quality of the pattern or its modification during the tests, particularly in specific areas such as the main cracks or previous marks on the face of the specimens. Small discontinuities in the full-field maps can usually be interpolated without introducing too much error, although this option has been disabled in this case to make them visible. These matching problems are perceptible in small areas of the GOM maps (Fig. 5a and c) but even more evident in the case of DICe (Fig. 5b and d), which presents discontinuities distributed all over the surface. These artefacts can locally distort the results, but the technique provides such a redundant number of measurements that it should not represent a problem to determine the global strength parameters of the specimen.

To compare the DICe and Aramis results in a quantitative manner, the displacement values at 5 points on the surface of specimen S1 (randomly selected from the 50 total checkpoints) are also plotted in Fig. 6. The figure shows a rather stable behaviour of the differences between the displacements measured with both systems, with a slight tendency to increase during the test in the case of the displacement perpendicular to the loading direction $(\mathrm{dX})$.

Considering the whole set of tests (Table 4) and their entire step count, the mean deviations between Aramis-3D and low-cost 2D-DIC measurements ranged between 0.01 


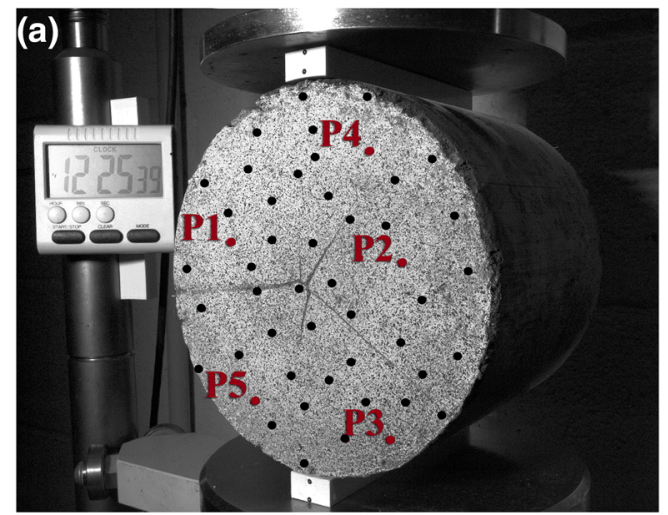

(c)

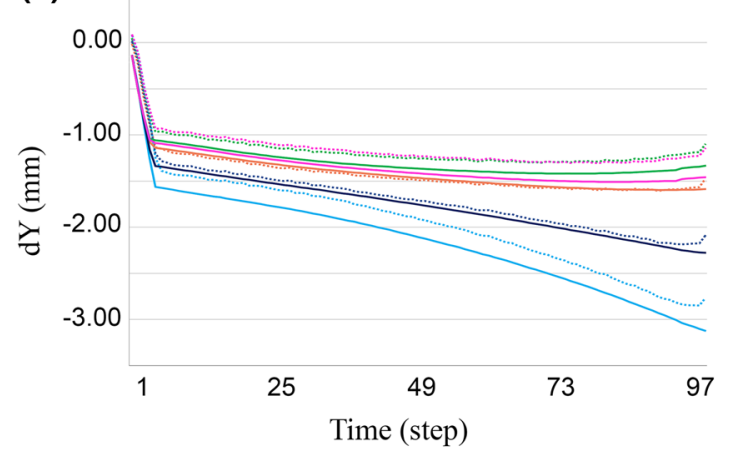

(b)

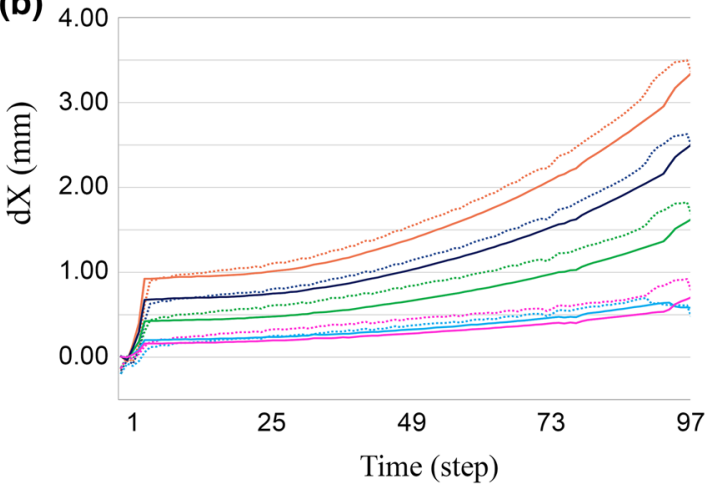

(d)
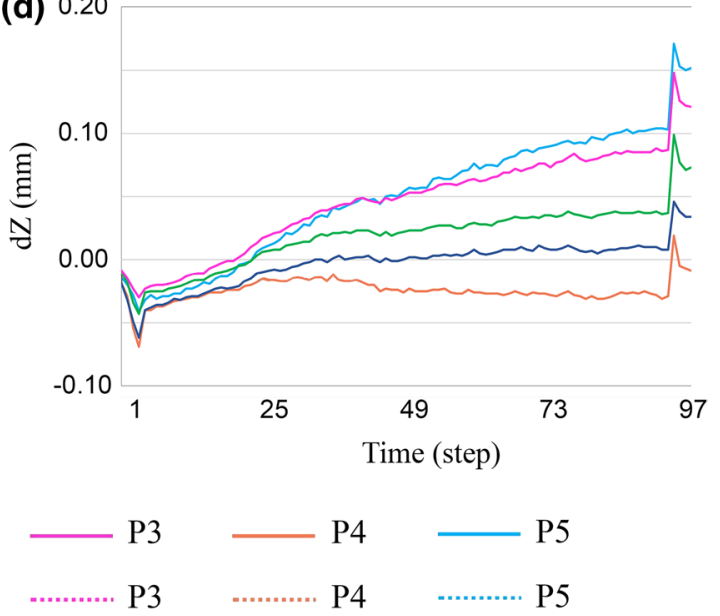

Fig. 6 Displacement analysis in BTS-S1. a Distribution of checkpoints over the face of the specimen. Magnitude of the displacements in the three components: $\mathbf{b} \mathrm{dX}, \mathbf{c} \mathrm{dY}$ and $\mathbf{d} \mathrm{dZ}$ obtained with Aramis 3D and DICe at 5 points on the specimen

and $0.16 \mathrm{~mm}$ in the direction perpendicular to loading $(\Delta \mathrm{X})$ and 0.03 and $0.09 \mathrm{~mm}$ in the loading direction $(\Delta \mathrm{Y})$. Even so, the comparative accuracy results between different tests should be read with caution, as some parameters of the optical systems (e.g., distance to object, image resolution, focal length) were configured independently for each test to keep the AOI constant. Moreover, other variables, such as the quality of the specimen's speckled patterns and the variable rate of loading, may have a strong influence on the results. However, the results provide a general idea of the order of magnitude of the errors using consumer-grade cameras for 2D-DIC. Given that the quality of the DIC measurements relies on numerous variables (e.g., the camera-object distance, the size of the sensor, the lens, the quality of the speckle pattern), it would be of interest to include a detailed description of all of these factors whenever this type of lowcost system is used. In particular, it has been shown that the distance to the object has a noticeable influence on the accuracy of the low-cost DIC. Therefore, it would also be good practice to express the DIC error as a 1: $d$ ratio, where $d$ is the distance to the object divided by the reported error.
This yields mean values of 1:5,100 for the experiments performed with the Sony ILCE 6000 at $525 \mathrm{~mm}$ (BTS-S1 and S2) and 1:9,800 for the Canon EOS550D setup at $350 \mathrm{~mm}$ (tests BTS-S3 and S4). It should be noted that this metric (i.e., relative error) has no scale and is simply a rough estimation of the true precision, which will vary within each image step. Expressing the relative precision is always a good practice when optical methods are used, as the errors are generally highly dependent on the distance to the object. However, the relative error does not replace the calculation of absolute errors from independent measurements.

As shown in the overall statistics for the BTS-S1 test (Table $4 \mathrm{a}$ ) based on the 50 checkpoints, both in-plane displacement components are dominant in terms of order of magnitude compared with the out-of-plane component. However, whilst a similar result applies to BTS-S2 (Table $4 \mathrm{~b}$ ), the last two tests (Table $4 \mathrm{c}$ and d) show much larger mean out-of-plane displacements (up to $4 \mathrm{~mm}$ ). The variability in $\mathrm{dZ}$ when performing BTS tests is always difficult to control, as it depends to a great extent on the way the fracture occurs, the possible buckling of the specimen and 
Table 4 Overall statistics per component in the four tests from 50 checkpoints on the surface

a) BTS-S1. 2D-DIC (with Sony ILCE $6000+$ DICe) vs. Aramis 3D

\begin{tabular}{|c|c|c|c|}
\hline & $\Delta \mathrm{X}\left(\mathrm{dX}_{\mathrm{DICe}}-\mathrm{dX}_{\mathrm{GOM}}\right)(\mathrm{mm})$ & $\Delta \mathrm{Y}\left(\mathrm{dY}_{\mathrm{DICe}^{-}}-\mathrm{dY}{ }_{\mathrm{GOM}}\right)(\mathrm{mm})$ & $\mathrm{dZ}_{\mathrm{GOM}}(\mathrm{mm})$ \\
\hline Mean & 0.0904 & 0.0891 & -0.0128 \\
\hline Median & 0.0943 & 0.0812 & -0.0020 \\
\hline SD & 0.1129 & 0.0924 & 0.0450 \\
\hline Max & 0.4550 & 0.7607 & 0.0810 \\
\hline Min & -0.4087 & -0.1178 & -0.3230 \\
\hline \multicolumn{4}{|c|}{ b) BTS-S2. 2D-DIC (with Sony ILCE $6000+$ DICe) vs. Aramis 3D } \\
\hline & $\Delta \mathrm{X}\left(\mathrm{dX}_{\mathrm{DICe}^{-\mathrm{d}}} \mathrm{X}_{\mathrm{GOM}}\right)(\mathrm{mm})$ & $\Delta \mathrm{Y}\left(\mathrm{dY}_{\mathrm{DICe}^{-\mathrm{dY}}}{ }_{\mathrm{GOM}}\right)(\mathrm{mm})$ & $\mathrm{dZ}_{\mathrm{GOM}}(\mathrm{mm})$ \\
\hline Mean & 0.1636 & 0.0619 & 0.0342 \\
\hline Median & 0.1700 & 0.0605 & 0.0360 \\
\hline SD & 0.0564 & 0.0196 & 0.0480 \\
\hline Max & 0.3355 & 0.1228 & 0.1790 \\
\hline Min & -0.0034 & -0.0080 & -0.2740 \\
\hline \multicolumn{4}{|c|}{ c) BTS-S3. 2D-DIC (with Canon 550D+DICe) vs. Aramis 3D } \\
\hline & $\Delta \mathrm{X}\left(\mathrm{dX}_{\mathrm{DICe}^{-\mathrm{dX}}}{ }_{\mathrm{GOM}}\right)(\mathrm{mm})$ & $\Delta \mathrm{Y}\left(\mathrm{dY}_{\mathrm{DICe}^{-\mathrm{dY}}}{ }_{\mathrm{GOM}}\right)(\mathrm{mm})$ & $\mathrm{dZ}_{\mathrm{GOM}}(\mathrm{mm})$ \\
\hline Mean & 0.0010 & -0.0580 & -0.3995 \\
\hline Median & 0.0028 & -0.0607 & -0.3820 \\
\hline SD & 0.0549 & 0.0342 & 0.1727 \\
\hline Max & 0.3809 & 0.0626 & 0.0070 \\
\hline Min & -0.1835 & -0.2117 & -0.9920 \\
\hline \multicolumn{4}{|c|}{ d) BTS-S4. 2D-DIC (with Canon 550D + DICe) vs. Aramis 3D } \\
\hline & $\Delta \mathrm{X}\left(\mathrm{dX}_{\mathrm{DICe}^{-}}-\mathrm{dX}{ }_{\mathrm{GOM}}\right)(\mathrm{mm})$ & $\Delta \mathrm{Y}\left(\mathrm{dY}_{\mathrm{DICe}^{-\mathrm{dY}}}{ }_{\mathrm{GOM}}\right)(\mathrm{mm})$ & $\mathrm{dZ}_{\mathrm{GOM}}(\mathrm{mm})$ \\
\hline Mean & 0.0475 & 0.0270 & -0.3344 \\
\hline Median & 0.0340 & -0.0287 & -0.3080 \\
\hline SD & 0.0937 & 0.2651 & 0.1505 \\
\hline Max & 0.5167 & 1.6050 & 0.0050 \\
\hline Min & -0.8830 & -0.4058 & -0.9170 \\
\hline
\end{tabular}

other factors. Even small variations in the initial positioning of the specimens or misaligned grips can lead to rotations or translations that will introduce significant differences in the measured magnitudes. In 2D-DIC, a slight misalignment of the optical axis of the camera with the normal to the specimen surface can also introduce a relative out-of-plane error, resulting in biased displacement data. Because the specimen is always assumed to be planar for out-of-plane motion in 2D-DIC, these displacements often represent the largest source of error in the planar components $[62,63]$.

In that sense, stereo-vision systems offer a clear advantage by allowing the joint analysis of the three spatial components. The analysis of checkpoints presented in Fig. 6d (BTS-S1) exemplifies the potential of 3D-DIC to capture even small movements and understand the results (in this case, the lower part of the specimen seems to have shifted forward over time until failure). However, the 3D-DIC system involves multiple correlation runs, including cross-camera subset matching, and requires the input of calibration data. According to previous studies, the eventual benefit of avoiding errors due to deviations from planarity can also introduce other error sources in the obtained displacement and strain fields [28]. This fact justifies to some extent why the results obtained in the measurement of the planar components do not show significant differences between Aramis-3D and the 2D-DIC low-cost system.

\section{Conclusions}

DIC technology has experienced rapid diffusion in recent years in a range of laboratory tests, such as the Brazilian indirect tensile strength test, proving to be a reliable alternative to traditional strain gauges. This experiment demonstrated that even a single consumer-grade camera with APS-C sensors ( size $<24 \times 16 \mathrm{~mm}$ ) that costs less than $\$ 1000$ can be useful 
in 2D-DIC setups for observing the fracture behaviour of brittle materials in experiments without high-strain rate events or high dynamic loading. The standardized BTS test of compacted soil specimens works satisfactorily with the use of such cameras because the test duration is in general reasonably long (with speeds of approximately $0.2-0.5 \mathrm{~mm} \cdot \mathrm{min}-1$ ), and the use of high-speed industrial cameras is not strictly required.

By using open-source software for processing image series, it is possible to extract basic qualitative DIC results as displacement maps that allow us to observe local behaviour phenomena during the BTS test due to material inhomogeneity, cracking, stress concentrations, plastic instabilities, and other characteristics. Moreover, the comparison of the results achieved in quantitative terms of accuracy shows little difference between free 2D-DIC and a professional 3D-DIC system with mean values of approximately $0.06 \mathrm{~mm}$ in planar components (i.e., 1:5,000-1:10,000 of relative error at setup distances). More specifically, the mean absolute deviations between Aramis-3D measurements and low-cost 2D-DIC were $0.08 \mathrm{~mm}$ in the direction perpendicular to loading $(\Delta \mathrm{X})$ and $0.06 \mathrm{~mm}$ in the loading direction $(\Delta \mathrm{Y})$. However, since outof-plane displacements caused by some effects that are hard to control (e.g., buckling, rotations, misalignment) reached mean values near $0.4 \mathrm{~mm}$ in some tests, they could have a significant influence on BTS test results. For that reason, further research should also address the implementation of stereo-DIC with consumer-grade cameras.

Supplementary Information The online version contains supplementary material available at https://doi.org/10.1007/s43452-021-00325-0.

Funding Open Access funding provided thanks to the CRUE-CSIC agreement with Springer Nature. This work was supported by the Strategic Researcher Cluster BioReDeS funded by the Regional Government Xunta de Galicia under the project Ref. ED431E 2018/09, by the Xunta de Galicia under the grant "Financial aid for the consolidation and structure of competitive units of investigation in the universities of the University Galician System (2020-22)" Ref. ED341B 2020/25 and by the Spanish Research Agency (AEI) in the frame of the programme Juan de la Cierva-Formación (Dr. Bastos) Ref. FJC2019-039743-I/ AEI/10.13039/501100011033.

\section{Declarations}

Conflict of interest The authors have no conflicts of interest to declare that are relevant to the content of this article.

Ethical approval This research does not concern experiments involving humans and/or animals and does not require the approval of the ethics committee.

Open Access This article is licensed under a Creative Commons Attribution 4.0 International License, which permits use, sharing, adaptation, distribution and reproduction in any medium or format, as long as you give appropriate credit to the original author(s) and the source, provide a link to the Creative Commons licence, and indicate if changes were made. The images or other third party material in this article are included in the article's Creative Commons licence, unless indicated otherwise in a credit line to the material. If material is not included in the article's Creative Commons licence and your intended use is not permitted by statutory regulation or exceeds the permitted use, you will need to obtain permission directly from the copyright holder. To view a copy of this licence, visit http://creativecommons.org/licenses/by/4.0/.

\section{References}

1. Fourmeau M, Gomon D, Vacher R, Hokka M, Kane A, Kuokkala V-T. Application of DIC technique for studies of Kuru granite rock under static and dynamic loading, procedia. Mater Sci. 2014;3:691-7. https://doi.org/10.1016/j.mspro.2014.06.114.

2. Mardoukhi A, Mardoukhi Y, Hokka M, Kuokkala VT. Effects of heat shock on the dynamic tensile behavior of granitic rocks. Rock Mech Rock Eng. 2017;50:1171-82. https://doi.org/10.1007/ s00603-017-1168-4.

3. Maruvanchery V, Kim E. Effects of water on rock fracture properties: studies of mode I fracture toughness, crack propagation velocity, and consumed energy in calcite-cemented sandstone. Geomech Eng. 2019;17:57-67. https://doi.org/10.12989/gae.2019. 17.1.057.

4. Stirling RA, Simpson DJ, Davie CT. The application of digital image correlation to Brazilian testing of sandstone. Int J Rock Mech Min Sci. 2013;60:1-11. https://doi.org/10.1016/j.ijrmms. 2012.12.026.

5. Mubaraki M, Abd-Elhady AA, Sallam HEDM. Mixed mode fracture toughness of recycled tire rubber-filled concrete for airfield rigid pavements. Int J Pavement Res Technol. 2013;6:8-14.

6. Wang P, Gao N, Ji K, Stewart L, Arson C. DEM analysis on the role of aggregates on concrete strength. Comput Geotech. 2020. https://doi.org/10.1016/j.compgeo.2019.103290.

7. Romeo E. Two-dimensional digital image correlation for asphalt mixture characterisation: interest and limitations. Road Mater Pavement Des. 2013. https://doi.org/10.1080/14680629.2013. 815128.

8. Akin ID, Likos WJ. Brazilian tensile strength testing of compacted clay. Geotech Test J. 2017;40:608-17. https://doi.org/10.1520/ GTJ20160180.

9. Stirling RA, Hughes P, Davie CT, Glendinning S. Tensile behaviour of unsaturated compacted clay soils-a direct assessment method. Appl Clay Sci. 2015;112-113:123-33. https://doi.org/ 10.1016/j.clay.2015.04.011.

10. Erarslan DJ, Williams N. Investigating the effect of cyclic loading on the indirect tensile strength of rocks. Rock Mech Rock Eng. 2012;45:327-40. https://doi.org/10.1007/s00603-011-0209-7.

11. Erarslan N, Alehossein H, Williams N. Tensile fracture strength of Brisbane tuff by static and cyclic loading tests. Rock Mech Rock Eng. 2014;47:1135-51. https://doi.org/10.1007/ s00603-013-0469-5.

12. Yang YW, Bhalla S, Wang C, Soh CK, Zhao J. Monitoring of rocks using smart sensors. Tunn Undergr Space Technol. 2007;22:206-21. https://doi.org/10.1016/j.tust.2006.04.004.

13. Belrhiti Y, Dupre JC, Pop O, Germaneau A, Doumalin P, Huger M, Chotard T. Combination of Brazilian test and digital image correlation for mechanical characterization of refractory materials. J Eur Ceram Soc. 2017;37:2285-93. https://doi.org/10.1016/j. jeurceramsoc.2016.12.032.

14. Sgambitterra E, Lamuta C, Candamano S, Pagnotta L. Brazilian disk test and digital image correlation: a methodology for the mechanical characterization of brittle materials. Mater Struct. 2018;51:1-17. https://doi.org/10.1617/s11527-018-1145-8.

15. Aliabadian Z, Zhao G, Russell AR. Crack development in transversely isotropic sandstone discs subjected to Brazilian tests 
observed using digital image correlation. Int J Rock Mech Min Sci. 2019;119:211-21. https://doi.org/10.1016/j.ijrmms.2019.04. 004.

16. Zhang H, Nath F, Parrikar PN, Mokhtari M. Analyzing the validity of Brazilian testing using DIC and numerical simulation techniques. Energies. 2020;13:1441.

17. Dutler N, Nejati M, Valley B, Amann F, Molinari G. On the link between fracture toughness, tensile strength, and fracture process zone in anisotropic rocks. Eng Fract Mech. 2018;201:56-79. https://doi.org/10.1016/j.engfracmech.2018.08.017.

18. Ravanelli R, Nascetti A, Di Rita M, Belloni V, Mattei D, Nisticó N, Crespi M. A new igital image correlation software for displacements field measurement in structural applications. Int Arch Photogramm Remote Sens Spatial Inf Sci. 2017;42:139-45. https://doi.org/10.5194/isprs-archi ves-XLII-4-W2-139-2017.

19. Gauvin C, Jullien D, Doumalin P, Dupré JC, Gril J. Image correlation to evaluate the influence of hygrothermal loading on wood. Strain. 2014;50:428-35. https://doi.org/10.1111/str. 12090.

20. Phillips N, Hassan GM, Dyskin A, Macnish C, Pasternak E. Digital image correlation to analyze nonlinear elastic behavior of materials. Proc Int Conf Image Process. 2018. https://doi.org/10. 1109/ICIP.2017.8297107.

21. Mojsilović N, Salmanpour AH. Masonry walls subjected to in-plane cyclic loading: application of digital image correlation for deformation field measurement. Int J Mason Res Innov. 2016;1:165-87. https://doi.org/10.1504/IJMRI.2016.077473.

22. Halding PS, Schmidt JW, Christensen CO. DIC-monitoring of full-scale concrete bridge using high-resolution wide-angle lens camera. In: Maintenance, safety, risk, management and life-cycle performance of bridges. CRC Press; 2018. https://doi.org/10.1201/ 9781315189390-203.

23. Quanjin M, Rejab MRM, Halim Q, Merzuki MNM, Darus MAH. Experimental investigation of the tensile test using digital image correlation (DIC) method. Mater Today Proc. 2020;27:757-63. https://doi.org/10.1016/j.matpr.2019.12.072.

24. Li C, Luo H, Pan B. High-throughput measurement of coefficient of thermal expansion using a high-resolution digital single-lens reflex camera and digital image correlation. Rev Sci Instrum. 2020. https://doi.org/10.1063/5.0013496.

25. Zhao $\mathrm{P}$, Zsaki AM, Nokken MR. Using digital image correlation to evaluate plastic shrinkage cracking in cement-based materials. Constr Build Mater. 2018;182:108-17. https://doi.org/10.1016/j. conbuildmat.2018.05.239.

26. Chen F, Chen X, Xie X, Feng X, Yang L. Full-field 3D measurement using multi-camera digital image correlation system. Opt Lasers Eng. 2013;51:1044-52. https://doi.org/10.1016/j.optla seng.2013.03.001.

27. Yu L, Lubineau G. Modeling of systematic errors in stereo-digital image correlation due to camera self-heating. Sci Rep. 2019;9:115. https://doi.org/10.1038/s41598-019-43019-7.

28. Wang P, Guo X, Sang Y, Shao L, Yin Z, Wang Y. Measurement of local and volumetric deformation in geotechnical triaxial testing using 3D-digital image correlation and a subpixel edge detection algorithm. Acta Geotech. 2020;15:2891-904. https://doi.org/10. 1007/s11440-020-00975-z.

29. White TG, Patten JRW, Wan KH, Pullen AD, Chapman DJ, Eakins DE. A single camera three-dimensional digital image correlation system for the study of adiabatic shear bands. Strain. 2017;53:111. https://doi.org/10.1111/str.12226.

30. Chi Y, Yu L, Pan B. Low-cost, portable, robust and high-resolution single-camera stereo-DIC system and its application in high-temperature deformation measurements. Opt Lasers Eng. 2018;104:141-8. https://doi.org/10.1016/j.optlaseng.2017.09.020.
31. Yu L, Tao R, Lubineau G. Accurate 3D shape, displacement and deformation measurement using a smartphone. Sensors. 2019. https://doi.org/10.3390/s19030719.

32. Pan B, Yu L, Zhang Q. Review of single-camera stereo-digital image correlation techniques for full-field 3D shape and deformation measurement. Sci China Technol Sci. 2018;61:2-20. https:// doi.org/10.1007/s11431-017-9090-x.

33. Solav D, Moerman KM, Jaeger AM, Genovese K, Herr HM. MultiDIC: an open-source toolbox for multi-view 3D digital image correlation. IEEE Access. 2018;6:30520-35. https://doi. org/10.1109/ACCESS.2018.2843725.

34. Blaber J, Adair B, Antoniou A. Ncorr: open-source 2D digital image correlation Matlab software. Exp Mech. 2015;55:1105-22. https://doi.org/10.1007/s11340-015-0009-1.

35. Olufsen SN, Andersen ME, Fagerholt E. $\mu$ DIC: an open-source toolkit for digital image correlation. SoftwareX. 2020;11: 100391. https://doi.org/10.1016/j.softx.2019.100391.

36. Belloni V, Ravanelli R, Nascetti A, Di Rita M, Mattei D, Crespi M. Py2dic: a new free and open source software for displacement and strain measurements in the field of experimental mechanics. Sensors (Switzerland). 2019;19:1-19. https://doi.org/10.3390/ s19183832.

37. Belloni V, Ravanelli R, Nascetti A, Di Rita M, Mattei D, Crespi M. Digital image correlation from commercial to FOS software: a mature technique for full-field displacement measurements. Int Arch Photogramm Remote Sens Spatial Inf Sci. 2018;42:91-5. https://doi.org/10.5194/isprs-archives-XLII-2-91-2018.

38. Lunt D, Thomas R, Roy M, Duff J, Atkinson M, Frankel P, Preuss $\mathrm{M}$, da Fonseca JQ. Comparison of sub-grain scale digital image correlation calculated using commercial and open-source software packages. Mater Charact. 2020. https://doi.org/10.1016/j.matchar. 2020.110271.

39. ISRM. The ISRM suggested methods for rock characterization, testing and monitoring: 2007-2014. Cham: Springer; 2015. https://doi.org/10.1007/978-3-319-07713-0.

40. ASTM, D3967-16 (2016) Standard test method for splitting tensile strength of intact rock core specimens. ASTM International.

41. Perras MA, Diederichs MS. A review of the tensile strength of rock: concepts and testing. Geotech Geol Eng. 2014;32:525-46. https://doi.org/10.1007/s10706-014-9732-0.

42. UNE-EN 13286-2 (2011) Unbound and hydraulically bound mixtures-part 2: test methods for laboratory reference density and water content-proctor compaction. UNE standards.

43. Celeiro M, Lamas JP, Arcas R, Lores M. Antioxidants profiling of by-products from Eucalyptus Greenboards Manufacture. Antioxidants. 2019;8:263. https://doi.org/10.3390/antiox8080263.

44. Zhang T, Liu S, Zhan H, Ma C, Cai G. Durability of silty soil stabilized with recycled lignin for sustainable engineering materials. J Clean Prod. 2020;248: 119293. https://doi.org/10.1016/j.jclepro. 2019.119293.

45. Yang B, Zhang Y, Ceylan H, Kim S, Gopalakrishnan K. Assessment of soils stabilized with lignin-based byproducts. Transp Geotech. 2018;17:122-32. https://doi.org/10.1016/j.trgeo.2018. 10.005 .

46. Sharafisafa M, Shen L, Xu Q. Characterisation of mechanical behaviour of 3D printed rock-like material with digital image correlation. Int J Rock Mech Min Sci. 2018;112:122-38. https:// doi.org/10.1016/j.ijrmms.2018.10.012.

47. Reu P. The art and application of DIC. Stereo-rig design: stereoangle selection-part 4. Exp Tech. 2013;37:1-2.

48. IDICS. A good practices guide for digital image correlation. Int Digit Image Correl Soc. 2018. https://doi.org/10.32720/idics/gpg. ed1.

49. Sciuti VF, Canto RB, Neggers J, Hild F. On the benefits of correcting brightness and contrast in global digital image correlation: Monitoring cracks during curing and drying of a refractory 
castable. Opt Lasers Eng. 2021;136: 106316. https://doi.org/10. 1016/j.optlaseng.2020.106316.

50. Zhang Z. A flexible new technique for camera calibration. IEEE Trans Pattern Anal Mach Intell. 2000;22:1330-4. https://doi.org/ 10.1109/34.888718.

51. Chen L-C, Chang C-Y, Lee W-C, Ma C-C. Full-field measurement of deformation and vibration using digital image correlation. Smart Sci. 2015;3:80-6. https://doi.org/10.1080/23080477.2015. 11665640.

52. Kasprzak B, Pękala J, Stępień A, Świerczyński Z. Metrology and measurement systems. Architecture. 2010. https://doi.org/10. 1515/mms-2016-0028. Brought.

53. Zhong FQ, Indurkar PP, Quan CG. Three-dimensional digital image correlation with improved efficiency and accuracy. Meas J Int Meas Confed. 2018;128:23-33. https://doi.org/10.1016/j. measurement.2018.06.022.

54. Hartley R, Zisserman A. Multiple view geometry in computer vision. Cambridge University Press; 2005.

55. Balcaen R, Reu PL, Lava P, Debruyne D. Stereo-DIC uncertainty quantification based on simulated images. Exp Mech. 2017;57:939-51. https://doi.org/10.1007/s11340-017-0288-9.

56. Sutton MA, Matta F, Rizos D, Ghorbani R, Rajan S, Mollenhauer $\mathrm{DH}$, Schreier HW, Lasprilla AO. Recent progress in digital image correlation: background and developments since the $2013 \mathrm{~W}$ M Murray Lecture. Exp Mech. 2017;57:1-30. https://doi.org/10. 1007/s11340-016-0233-3.

57. Ahrens J, Geveci B, Law C (2005) ParaView: an end-user tool for large data visualization, Tech Rep LA-UR-03-1. The visualization handbook. 2005; 717:8:1-17.
58. Hu X, Xie Z, Liu F. Estimating gray intensities for saturated speckle to improve the measurement accuracy of digital image correlation. Opt Lasers Eng. 2021;139: 106510. https://doi.org/ 10.1016/j.optlaseng.2020.106510.

59. Dong YL, Pan B. A review of speckle pattern fabrication and assessment for digital image correlation. Exp Mech. 2017;57:1161-81. https://doi.org/10.1007/s11340-017-0283-1.

60. UNE-EN 13286-2 (2003) Unbound and hydraulically bound mixtures part 42: test method for the determination of the indirect tensile strength of hydraulically bound mixtures. UNE standards.

61. He W, Chen K, Hayatdavoudi A, Huang P, Sawant K, Zhang C. Incorporating the effects of elemental concentrations on rock tensile failure. Int J Rock Mech Min Sci. 2019;123: 104062. https:// doi.org/10.1016/j.ijrmms.2019.104062.

62. Sutton MA, Yan JH, Tiwari V, Schreier HW, Orteu JJ. The effect of out-of-plane motion on 2D and 3D digital image correlation measurements. Opt Lasers Eng. 2008;46:746-57. https://doi.org/ 10.1016/j.optlaseng.2008.05.005.

63. Siegmann P, Felipe-Sesé L, Díaz FA. An alternative approach for improving DIC by using out-of-plane displacement information. Opt Lasers Eng. 2020;128: 105996. https://doi.org/10.1016/j.optla seng.2019.105996.

Publisher's Note Springer Nature remains neutral with regard to jurisdictional claims in published maps and institutional affiliations. 